\title{
miR-483 is down-regulated in gastric cancer and suppresses cell proliferation, invasion and protein $\mathrm{O}-$ GlcNAcylation by targeting OGT
}

\author{
F. Y. YU, C. Y. ZHOU*, Y. B. LIU, B. WANG, L. MAO, Y. LI \\ Department of Radiation Oncology, Yueyang Second People’s Hospital, Yueyang 414000, China \\ *Correspondence: zhouchuanyiyy@163.com
}

Received June 8, 2017 / Accepted August 16, 2017

\begin{abstract}
MicroRNAs (miRNAs) are involved in the gastric carcinogenesis and progression. Here, we confirmed that miR- 483 was frequently decreased in gastric cancer patients. The expression levels of miR- 483 were negatively correlated with tumor stage, node metastasis and stromal invasion. Log-rank tests demonstrated that low expression of miR-483 was strongly correlated with poor overall survival in patients with gastric cancer. Moreover, ectopic expression of miR-483 remarkably suppressed gastric cancer cell proliferation by enhancing cell apoptosis and significantly inhibited the invasion of gastric cancer cells, while low expression of miR-483 exhibited the opposite effect. Bioinformatics analysis revealed that OGT was a potential target of miR-483, and miR-483 inhibited the expression level of OGT mRNA by direct binding to its 3'-untranslated region (3'UTR). Expression of miR-483 was negatively correlated with OGT in gastric cancer tissues. In addition, modulation of miR-483 expression could affect the global cellular protein O-GlcNAcylation in gastric cancer cells. Furthermore, silencing of OGT counteracted the effects of miR-483 repression, while its overexpression reversed tumor inhibitory effects of miR-483. In conclusion, our study revealed that miR-483 functions as a tumor suppressor by inhibiting proliferation, invasion and protein O-GlcNAcylation of gastric cancer via targeting OGT, and that miR-483 may serve as a prognostic or therapeutic target for gastric cancer.
\end{abstract}

Key words: gastric cancer, OGT, miR-483, proliferation, invasion

Gastric cancer is the fourth most common malignancy and ranks the second in the cause of cancer-related deaths worldwide [1]. Despite improvements in the diagnosis and treatment of this cancer, the long-term survival of gastric cancer patients has been only modestly improved over the past decade [2]. For a lack of specific early symptoms, it is often diagnosed at an advanced stage and curative chance is lost with extremely poor prognosis. It has been indicated that the pathogenesis of gastric cancer is multifactorial and the regulatory mechanism involved in the development of gastric cancer is not well understood. Therefore, it is critical to investigate the mechanisms underlying the development and progression of gastric cancer, which provides crucial insights into discovery of novel diagnostic biomarkers and identification of new therapeutic targets for gastric cancer patients.

MicroRNAs are a family of small, non-coding RNAs of approximately 22 nucleotides in length that negatively regulate gene expression mainly through their effects on productive translation and mRNA stability [3]. Accumulating evidence has confirmed that miRNAs are closely related with the occurrence, invasion and metastasis of various human cancers and act either as oncogenes or tumor suppressors owing to their critical roles in diverse biological functions, such as cell proliferation, apoptosis, as well as differentiation [4]. To date, large number of miRNAs has been implicated in the development and progression of gastric cancer [5-7]. MiR-483, also designated as miR-483-3p [8,9], has consistently been indicated in gastric cancer [10-13], and a number of studies have shown that miR-483 is frequently altered in a large variety of cancers, and that the functional role of this miRNA is extremely complex as it may act as an oncogenic or a tumor-suppressive miRNA depending on the cellular contexts [14-17]. Given the complexity of its functionality, it would be of interest to investigate the functional role and the exact mechanisms of miR-483 involved in gastric carcinogenesis.

In this study, we demonstrate that miR-483 is significantly downregulated in gastric cancer tissue and cell lines. Ectopic expression of miR-483 suppressed cell proliferation and invasion via decreasing protein O-GlcNAcylation in cancer cells by directly targeting OGT, and miR-483 expression is 
negatively associated with OGT expression in gastric cancer. These findings indicate that the miR-483/OGT axis plays a crucial role in gastric cancer progression through acting as an important regulator of O-GlcNAcylation in cancer cells.

\section{Patients and methods}

Clinical samples, cell lines and transfection. Gastric cancer tissues and corresponding adjacent normal tissues were obtained from 54 patients with gastric cancer who received surgery at the Wuhan Tumor Hospital, Wuhan, China. Samples were obtained with informed consent, and the study was approved by local institutional review boards on human subject research and in accordance with the Declaration of Helsinki. All the histological diagnoses for gastric cancer and normal tissues were reviewed and recognized independently by two pathologists. Clinicopathologic information about the patient samples used in this study is described in Table 1. GES-1 (human immortal gastric epithelial cell line) and five human gastric cancer cell lines SGC7901, MKN45, MKN28, AGS and SNU16 were purchased from Type Culture Collection of the Chinese Academy of Sciences (Shanghai, China) and cultured according to the provider's instruction. Transfection was performed when cells were grown to $70 \%$ confluence, using the Lipofectamine 2000 (Invitrogen, Carlsbad, CA, USA) according to the manufacturer's instructions.

MicroRNA and plasmid construction. miR-483 mimics or inhibitor which functionally inhibits endogenous miR-483 by competitively binding the seed sequence with target mRNA, and corresponding negative control were purchased from RiboBio (Guangzhou, China). OGT-specific siRNA and negative control were obtained from Ambion (Shanghai, China). OGT cDNA was subcloned into pcDNA3.1 to construct the OGT expression plasmid. For luciferase reporter, the 3'UTR of OGT containing the putative binding sites for miR-483 was amplified by PCR from human genomic DNA using the following forward primer: 5'-GTCATCTAGATCCTTTTCTTCCCTGACCCCCATAC-3' and the reverse primer: 5'-GTCATCTAGATCACTTTTGGAAGCAGCAT-3', both of which contain a XbaI restriction site. The PCR product was digested with $\mathrm{XbaI}$ and subcloned into pGL3 luciferase reporter plasmid (Promega, Madison, WI, USA). Mutations in the miR-483 binding site of OGT 3'UTR were produced by the QuikChange Site-Directed Mutagenesis Kit (Stratagene, La Jolla, CA, USA). Constructs were confirmed by sequencing.

RNA extraction and qRT-PCR. Total RNA and miRNA were extracted from tissues and cells using RNeasy Mini and miRNeasy Mini Kits (Qiagen, Valencia, CA, USA) according to the manufacturer's instructions. For examination of miR-483 expression, stem-loop RT-PCR was performed as described [18]. Expression of U6 was used as an endogenous control. Real-time PCR was performed using FastStart Universal SYBR Green Master kit (Roche Diagnostics, Mannhelm, Germany) and analyzed with an Applied Biosys- tems 7900 Real-Time PCR System. The PCR primers used were as follows: OGT, 5'-ACTGCAGGAAGCTCTGATGC-3' and 5'-ATCCTTATGAATGGAAGCCAG-3'; and GAPDH, 5'ATGTCGTGGAGTCTACTGGC-3' and 5'-TGACCTTGCCCACAGCCTTG-3'. Fold changes in expression were calculated. The $2^{-\Delta \Delta C t}$ method was used to calculate the foldchange of the RNA expression of one sample compared to the calibration sample [19]. All the qRT-PCRs were run in triplicate.

Cell proliferation, cell cycle analysis and apoptosis assay. Cell proliferation was monitored by MTT assay as described [18]. For cell cycle assay, cell pellets harvested by centrifugation were washed twice with ice-cold PBS and fixed with ice-cold $70 \%$ ethanol for $48 \mathrm{~h}$ at $4{ }^{\circ} \mathrm{C}$. The fixed cells were rehydrated in PBS and subjected to PI/RNase staining followed by fluorescence-activated cell sorter scan (FACS) analysis. For the cell apoptosis analysis, cells with different treatment were seeded in six-well plates at $3 \times 10^{5}$ cells per well in the absence of FBS for $48 \mathrm{~h}$. Then, cells were harvested and labeled with FITC-conjugated Annexin V and propidium iodide (BD Pharmingen) following the manufacturer's instructions. The apoptotic cells were measured by FACS.

Transwell invasion assay. Cell invasion assay was performed with Matrigel-coated transwell cell culture chambers (Millipore, Billerica, MA, USA). Aliquots of cells $\left(1 \times 10^{5}\right)$ were placed into upper chambers coated with $150 \mathrm{mg}$

Table 1. Correlations of miR-483 expression with clinicopathological parameters of gastric cancer patients.

\begin{tabular}{|c|c|c|c|}
\hline \multirow[b]{2}{*}{ Clinicopathological parameters } & \multicolumn{2}{|c|}{ miR-483 expression } & \multirow[b]{2}{*}{ p-value } \\
\hline & $\begin{array}{c}\text { Low } \\
(\mathrm{n}=27)\end{array}$ & $\begin{array}{c}\text { High } \\
(\mathbf{n}=27)\end{array}$ & \\
\hline \multicolumn{4}{|l|}{ Gender } \\
\hline Male & 12 & 7 & \\
\hline Female & 15 & 20 & 0.154 \\
\hline \multicolumn{4}{|l|}{ Age (years) } \\
\hline$\leq 60$ & 11 & 15 & \\
\hline$>60$ & 16 & 12 & 0.275 \\
\hline \multicolumn{4}{|l|}{ AJCC stage } \\
\hline I/II & 7 & 19 & \\
\hline III/IV & 20 & 8 & $0.001^{*}$ \\
\hline \multicolumn{4}{|l|}{ Tumor size $(\mathrm{cm})$} \\
\hline$<5$ & 13 & 12 & \\
\hline$\geq 5$ & 14 & 15 & 0.785 \\
\hline \multicolumn{4}{|l|}{ Lymph node metastasis } \\
\hline Negative & 6 & 17 & \\
\hline Positive & 21 & 10 & $0.002^{*}$ \\
\hline \multicolumn{4}{|l|}{ Invasion depth } \\
\hline Without Infiltration into Serous layer & 5 & 18 & \\
\hline Infiltration into Serous layer & 22 & 9 & $0.0006^{*}$ \\
\hline \multicolumn{4}{|l|}{ Vascular invasion } \\
\hline Negative & 17 & 15 & \\
\hline Positive & 10 & 12 & 0.156 \\
\hline
\end{tabular}

${ }^{*}$ Statistically significant difference $(\mathrm{p}<0.05)$ 
Matrigel (BD Biosciences, Bedford, MD, USA). The lower chambers were filled with DMEM containing $10 \%$ FBS. After incubation at $37^{\circ} \mathrm{C}$ for $24 \mathrm{~h}$, cells remaining on the upper surface of the membrane were removed. Cells on the lower surface of the membrane were fixed and stained with crystal violet. The invaded cells were counted at $\times 200$ magnification from five random fields of each filter. The assays were performed in triplicate and were repeated three times.

Dual-luciferase assay. For the dual-luciferase assay, HEK293 cells in a 96-well plate were transfected with 50nM miR-483 or miR-NC. The cells were then co-transfected with $0.2 \mathrm{mg} / \mathrm{ml}$ of vector with the wild-type or mutant $3^{\prime}$ UTR of OGT gene. After $48 \mathrm{~h}$, luciferase activity was measured with the Dual-Luciferase Reporter Assay System (Promega). Firefly luciferase activity was then normalized to the corresponding Renilla luciferase activity. Luciferase assays were performed in quadruplicate and repeated in three independent experiments.

Western blot. Cells were lysed in RIPA buffer in the presence of Protease Inhibitor Cocktail (Pierce, Rockford, USA). Protein was quantified by the BCA protein assay kit (Beyotime, Haimen, China). Equal amounts of protein were separated by SDS-PAGE, transferred onto PVDF membranes (Bio-Rad, Hercules, CA, USA) and blocked for $1 \mathrm{~h}$ at room temperature. Membranes were probed with primary antibodies against OGT, O-GlcNAc and GAPDH (Santa Cruz Biotechnology, Santa Cruz, CA, USA) at $4{ }^{\circ} \mathrm{C}$ overnight followed by incubation with HRP-conjugated secondary antibodies. Blots were detected using an ECL detection system.

Statistical analysis. All statistical analyses were performed using SPSS16.0 software (SPSS, Chicago, IL, USA). Experiments were repeated independently at least three times, and the results are expressed as mean $\pm \mathrm{SD}$. The correlation between miR-483 and OGT was analyzed using Spearman's correlation test. Statistical differences between groups were evaluated using Student's paired two-tailed t-test. A p $<0.05$ was considered statistically significant.

\section{Results}

MiR-483 is downregulated in gastric cancer. Several microarray-based studies indicated that miR- 483 was likely downregulated in gastric cancer. To further validate the aberrant expression of miR-483 in gastric cancer, we tested the expression of miR-483 in 54 pairs of gastric cancer tissues and their matched adjacent non-tumor tissues by qRT-PCR. We found that miR-483 expression was significantly decreased in gastric cancers than that of their non-tumor counterparts (Figure 1A, Figure S1). Next, the correlation between miR-483 expression and clinicopathological parameters was

Figure 1. miR-483 is decreased in gastric cancer tissues and cell lines and is negatively correlated with poor prognosis of gastric cancer patients. (A) The mean expression level of miR-483 in gastric cancer tissues was significantly lower than that in pair-matched adjacent non-cancerous gastric tissues. (B) Kaplan-Meier curves of the overall survivals of 54 gastric cancer patients were scored as low expression level (below the median value, $n=27)$ and high expression level (above the median value, $n=27$ ) according to the miR-483 expression. The miR-483 downregulation was significantly correlated with an overall shorter survival. (C) miR483 was significantly decreased in five gastric cancer cell lines compared with that in GES- 1 cell. ${ }^{*} \mathrm{p}<0.05,{ }^{* *} \mathrm{p}<0.01$ compared with

A

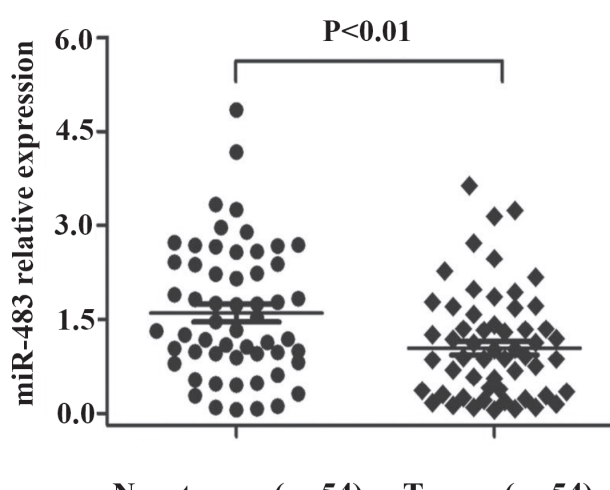

Non-tumor $(n=54) \quad$ Tumor $(n=54)$

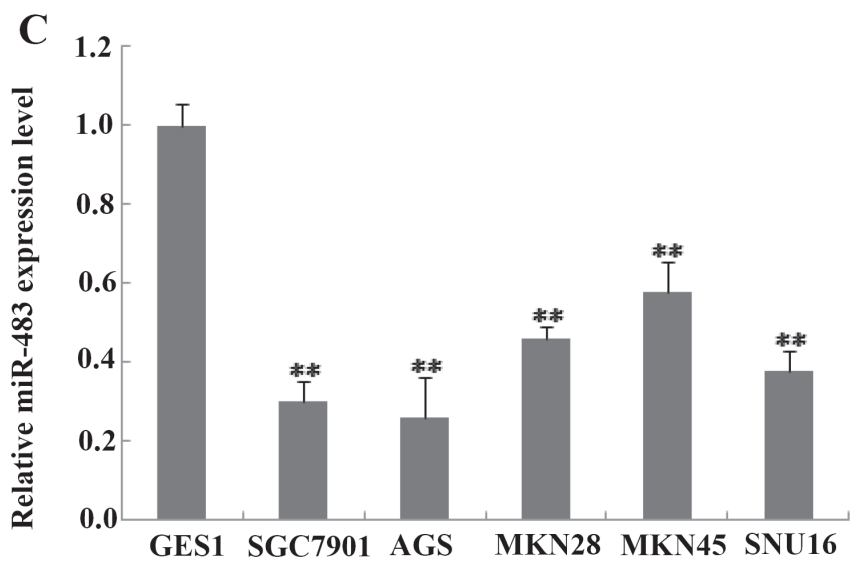

B

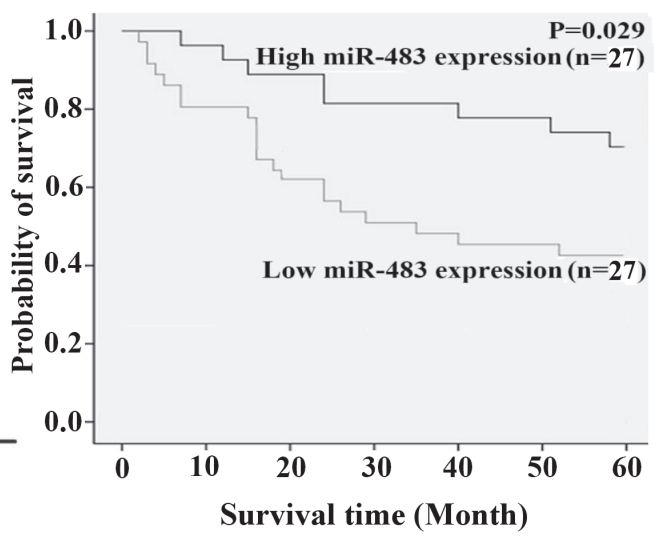
control group. 
further analyzed in 54 gastric cancer patients. Patients were divided into high and low expression group by the median expression level of miR-483. The statistical analysis revealed that miR-483 expression level was negatively correlated with the tumor stage, node metastasis and deep invasion while no significant correlation was observed in other parameters (Table 1). Kaplan-Meier survival analysis indicated that the patients with a low miR-483 expression had shorter overall survival times than those with a high miR-483 expression (Figure 1B). Then, we further examined the expression of
miR-483 in five different human ovarian cancer cell lines as well as human gastric normal epithelial cell line GES-1. The results indicated a significant downregulation in expression of miR-483 in gastric cancer lines as compared to GES-1 (Figure 1C). MKN45 and AGS cells exhibited the highest and lowest expression of miR-483 among gastric cancer cell lines, respectively, and were selected for further studies. These results indicate that miR-483 is downregulated in gastric cancer and low miR-483 expression is indicative of poor outcomes.
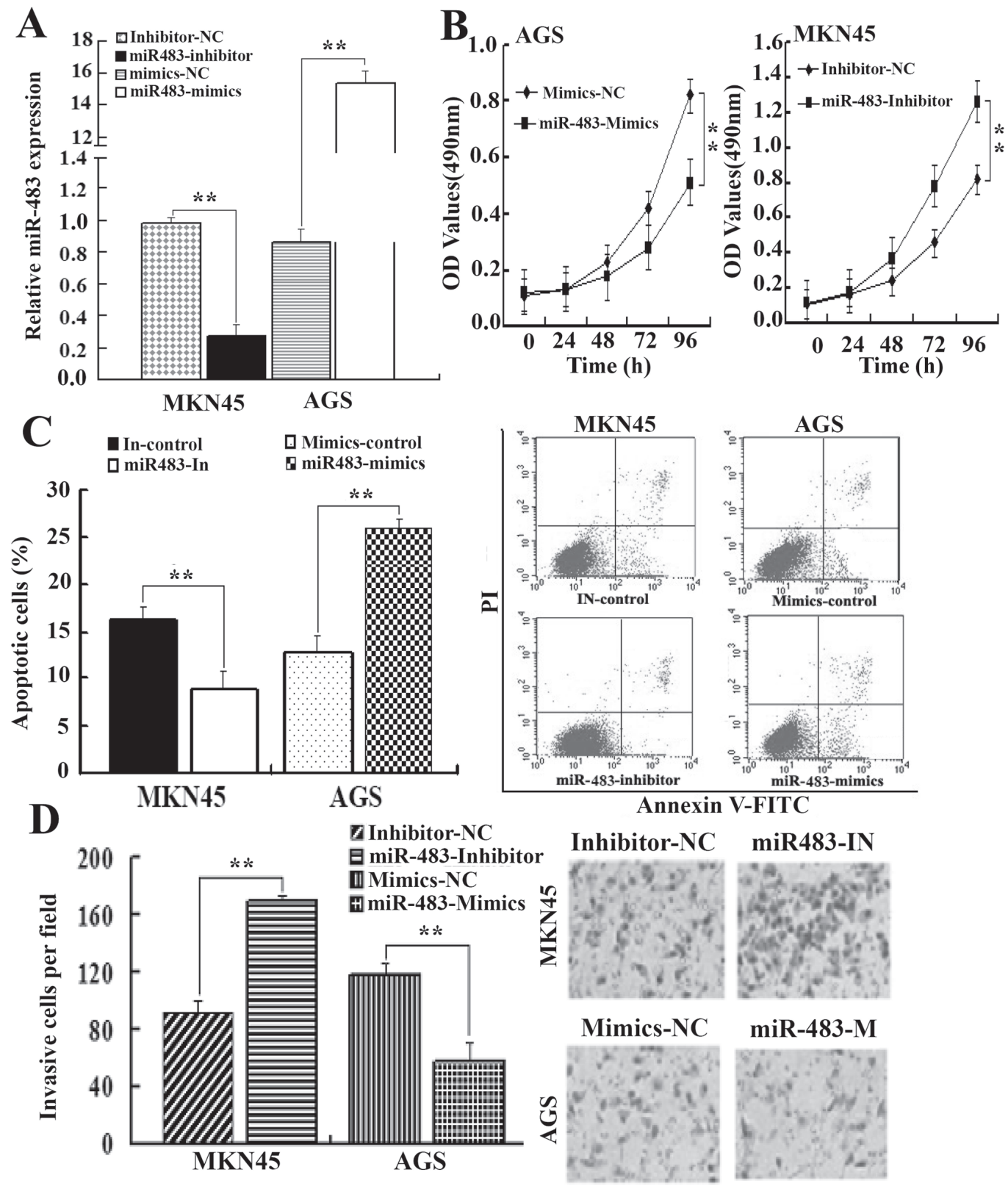

Figure 2. miR-483 suppresses gastric cancer cell proliferation and invasion. (A) qRT-PCR analysis of miR-483 levels in MKN45 and AGS cells transfected with miR-483 inhibitor and miR-483 mimics, respectively. (B) Determination of MKN45 and AGS cells proliferation with MTT assay. (C) Determination of MKN45 and AGS apoptosis with Flow cytometry. (D) Determination of MKN45 and AGS cells invasion with Transwell invasion assay. ${ }^{*} \mathrm{p}<0.05,{ }^{* *} \mathrm{p}<0.01$ compared with control group. 
miR-483 inhibits gastric cancer cell growth and invasion in vitro. To investigate the functions of miR-483 in gastric cancer, we performed knockdown and overexpression experiments in gastric cancer cells. MKN45 cells expressing relatively high level of miR-483 and AGS cells expressing relatively low level of miR-483 were transfected with miR-483 inhibitor and miR-483 mimics, respectively, which decreased the level of miR-483 in MKN45 by 3.8 folds and increased that in AGS by 15.8 folds, as compared to the corresponding negative control (Figure 2A). MTT assay indicated that downregulation of miR-483 significantly increased cell growth of MKN45 cells while upregulation of miR-483 significantly decreased cell growth of AGS cells, as compared to the corresponding negative controls (Figure 2B). To further explore the mechanisms of miR-483 affecting gastric cancer cell proliferation, we analyzed the apoptosis and cell cycle in the treated AGS and MKN45 cells by flow cytometry. We found that the downregulation of miR-483 significantly decreased cell apoptosis rate of MKN45 cells while upregulation of miR-483 significantly increased cell apoptosis rate of AGS cells, as compared to the corresponding negative controls (Figure 2C). There were no significant changes in the cell cycle for both cell lines (data not shown). These results suggest that miR-483 induced gastric cancer cell growth inhibition, at least in part, caused by apoptosis.

To explore the role of miR-483 in the invasion of gastric cancer cells, in vitro invasion assays were performed. Matrigel Transwell assays showed that miR-483 markedly repressed the invasive ability of AGS cells while anti-miR-483 promoted this activity of MKN45 cells (Figure 2D).

OGT is a direct target of miR-483. To screen potential targets for miR-483, we used three bioinformatics algorithms: TargetScan, miRDB and miRanda. All of these approaches predicted OGT as a target of miR-483, and the 3'-UTR of OGT mRNA contains a highly conserved binding site from position 785 to 792 for miR-483 seed sequence (the core sequence that encompasses the first 2-8 bases of the mature miRNA, Figure 3A). To confirm whether OGT was the target of miR-483, we performed Western blot analysis. The level of OGT protein was significantly decreased by miR-483 overexpression in AGS cells but significantly increased by silencing of miR-483 in MKN45 cells (Figure 3B). To examine whether the regulations described above in gastric cancer cell lines are also clinically relevant, we further tested the expression of OGT mRNA in 54 pairs of gastric cancer tissues and their matched adjacent normal tissues by qRT-PCR. The results show that OGT mRNA was significantly upregulated in gastric cancer tissues compared with the paired-adjacent normal tissues and negatively correlated with miR-483 level in gastric cancer tissues (Figure 3C, 3D). To further confirm whether OGT is a direct target gene of miR-483, we constructed luciferase reporter containing wild-type OGT 3'UTR with miR-483 binding site (WT) or containing the mutant 3'UTR (deletion of the putative miR-483 target site, MUT). Luciferase activity assay showed that miR-483 significantly inhibited the luciferase activity of OGT-3'UTRWT reporter, compared with control, but did not influence the mutant reporter in HEK293 cells (Figure 3E). Moreover, given the critical role of OGT in protein O-GlcNAcylation, we further investigated effect of miR-483 on the total O-GlcNAcylation levels in gastric cancer cells. We found that overexpression of miR-483 mimics led to a significantly decrease in O-GlcNAcylation level in AGS cells, whereas downregulation of miR-483 in MKN45 cells resulted in a significant increase in O-GlcNAcylation. Together, these data suggest that OGT is a direct target of miR-483 in gastric cancer.

OGT mediates miR-483-induced effects on gastric cancer growth, invasion and $\mathrm{O}-\mathrm{GlcNAcylation}$. To explore whether OGT is involved in the suppressor effects of miR-483 on gastric cancer cells, we co-transfected AGS cells with both miR-483 mimics and OGT-expressing vector, and MKN45 cells were co-transfected with both miR-483 inhibitor and siOGT. MTT and in vitro invasion assays showed that overexpression of OGT significantly reversed miR-483induced suppression of growth and invasion of AGS cells, while silencing of OGT significantly abrogated protumoral effects of miR-483 inhibitor on the growth and invasion of MKN45 cells (Figure 4A-C). Next, we explored whether miR-483 influencing gastric cancer cell O-GlcNAcylation was through targeting OGT. We found that overexpression of OGT markedly abrogated the miR-483 suppression effects on O-GlcNAcylation, while silencing OGT expression reversed the effect of miR-483 inhibitor on O-GlcNAcylation (Figure 4D). Collectively, these data indicate that miR-483 suppresses gastric cancer cell growth, invasion and O-GlcNacylation by directly targeting OGT.

\section{Discussion}

Several miRNAs microarray-based studies have indicated that miR-483 is down-regulated in gastric cancer [10-13]. However, the role, mechanism and clinical significance of miR-483 in gastric cancer have not been reported. In this study, we further confirmed that miR-483 was significantly down-regulated in both gastric cancer tissues and cell lines. We also found that miR-483 repressed gastric cancer cell growth by promotion of apoptosis, as well as inhibited gastric cancer cell invasion in vitro. Moreover, OGT was characterized as a functional target for miR-483.

Although dysregulation of miRNAs plays an important role in gastric cancer development, the relationship between aberrant miRNAs and clinical outcomes remains largely unknown. The survival analysis of this study showed that low expression of miR-483 was correlated with shorter survival time of gastric cancer patients. Our clinical data also indicated that down-regulation of miR-483 was significantly associated with advanced clinical stage, lymph node metastasis and deep stromal invasion, suggesting that miR-483 may play a critical role in progression of gastric cancer. It is becoming increasingly clear that chromosomal abnormalities 
and/or epigenetic events contribute to miRNAs deregulation. $\mathrm{Bi}$ et al. showed that down-regulation of miR-483 in multiple myeloma is related with its promoter hypermethylation [16]. It is of interest to further investigate the exact mechanisms of miR-483 down-regulation in gastric cancer.

Accumulated evidence indicated that the roles of miR-483 in cancer development were complicated, since miR-483 expression was up-regulated and functioned as an oncogene in pancreatic ductal adenocarcinoma and lung adenocarci- noma $[14,15]$, but significantly down-regulated and acted as a tumor-suppressor in multiple myeloma and squamous cell carcinomas $[16,17]$. In this study, we found that ectopic overexpression of miR-483 significantly suppressed proliferation and invasion in AGS cells, whereas downregulation of miR-483 promoted proliferation and invasion in MKN45 cells. These results suggest that miR-483 might function as a tumor suppressor miRNA in the progression of gastric carcinogenesis. Moreover, these data indicate that various

A has-miR-483 UUCUGCCCUCCUCUCCUCACU-

B
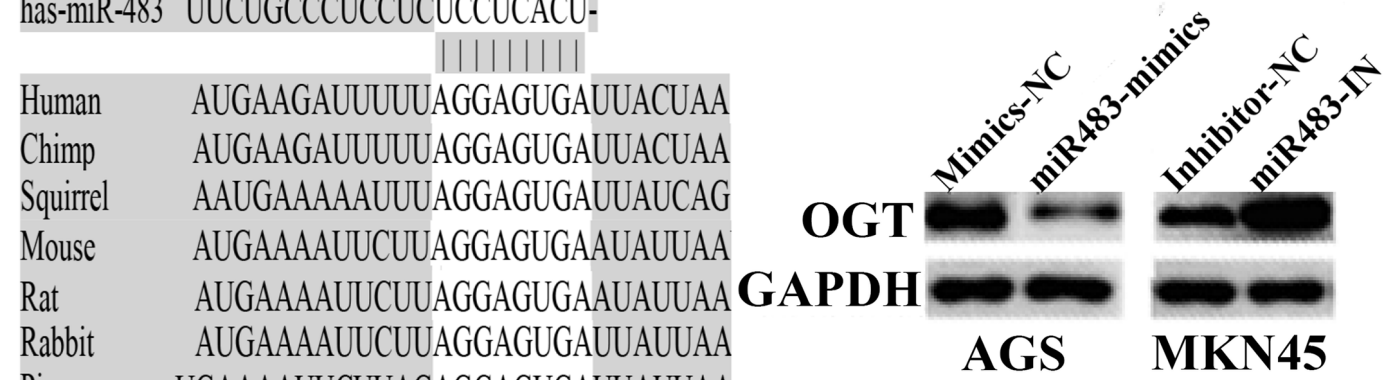

Pig UGAAAAUUCUUAGAGGAGUGAUUAUUAA
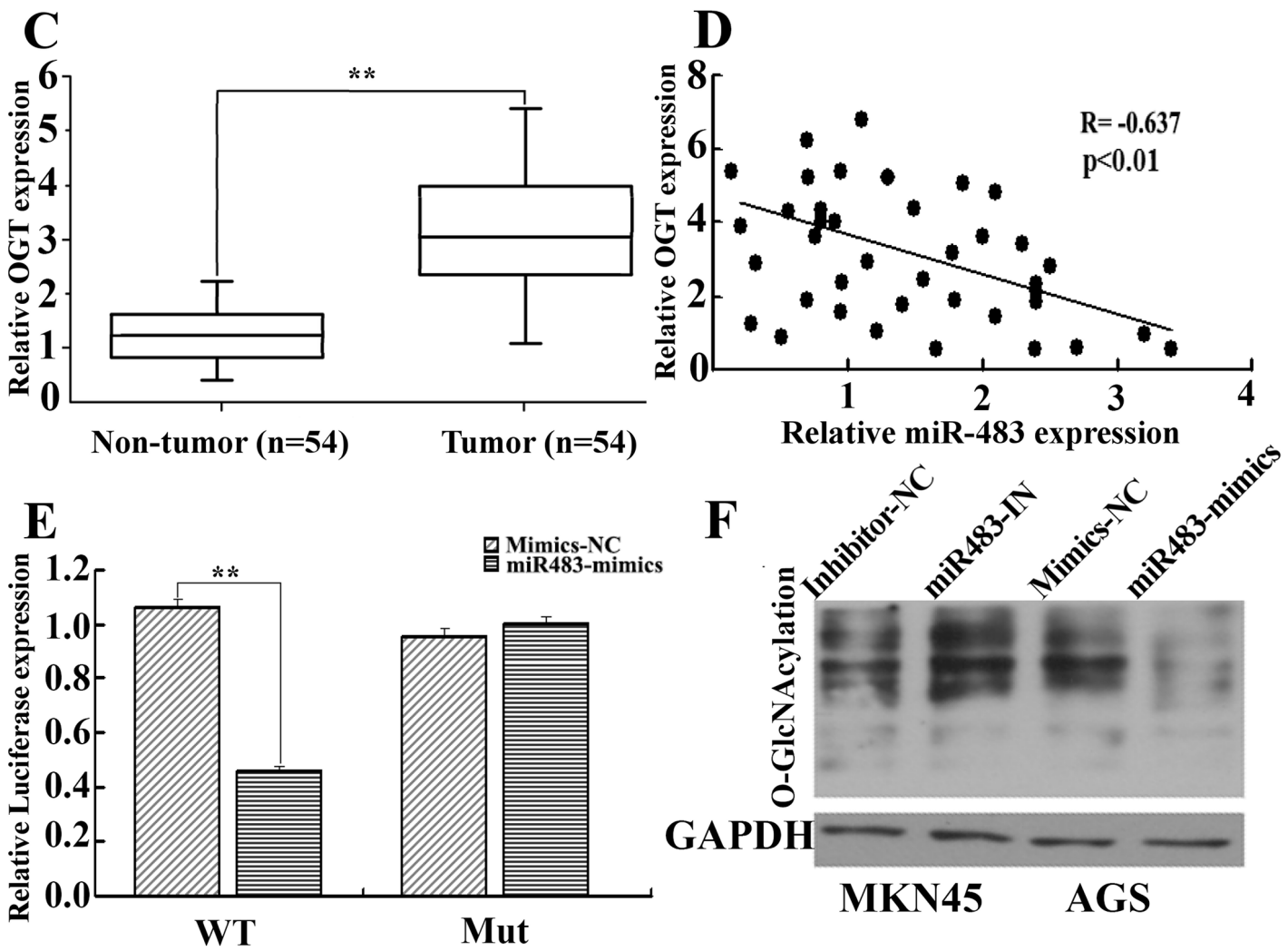

Figure 3. OGT is a direct target of miR-483. (A) A putative miR-483 binding site in 3'UTR region of OGT and interspecies conservation of seed matching sequences. Mutation was generated in the OGT 3'UTR by mutating seed matching sequence. (B) Protein level of OGT was detected by Western blot in MKN45 and AGS cells transfected with miR-483 inhibitor and miR-483 mimics along with corresponding controls, respectively. (C) OGT mRNA level was examined by qRT-PCR and it was remarkably increased in gastric cancer tissues. (D) OGT mRNA level was inversely correlated with miR483 level in gastric cancer tissues. (E) HEK293 cells were co-transfected with miR-483 and WT or Mut OGT 3'UTR luciferase reporter construct. (F) Determination of MKN45 and AGS cellular protein O-GlcNAcylation level by Western blot. ${ }^{*} \mathrm{p}<0.05,{ }^{* *} \mathrm{p}<0.01$ compared with control group. 
functions of miR-483 in different cancers are dependent on the cancer type and cellular context.

MiRNAs were reported to regulate gene expression by binding to the 3'-UTR of target mRNAs and either repressing translation or inducing degradation of mRNAs. In this study, we confirmed that miR-483 suppresses the expression of OGT in gastric cancer cells and luciferase reporter assay validated that OGT is a direct target of miR-483. Furthermore, we found that OGT expression was greatly upregulated in gastric cancer tissues and our correlation assay indicated that expression of miR-483 are negatively associated with those of OGT mRNA in gastric cancer tissues. Increased aerobic glycolysis in cancer cells is a universal feature of cancer progression, which increases glucose uptake and metabolic intermediates for macromolecule biosynthesis to support rapid cell growth $[20,21]$. Accumulating evidence suggests that O-linked- $\beta$ $\mathrm{N}$-acetylglucosamine modification may play a crucial role in tumorigenesis and redirection of cancer metabolism to

\section{$\mathbf{A}$}

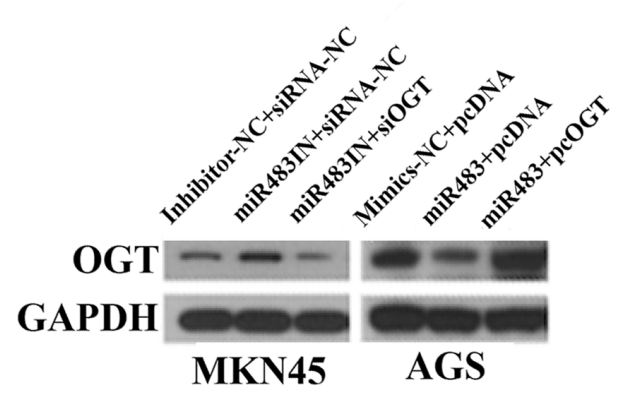

C

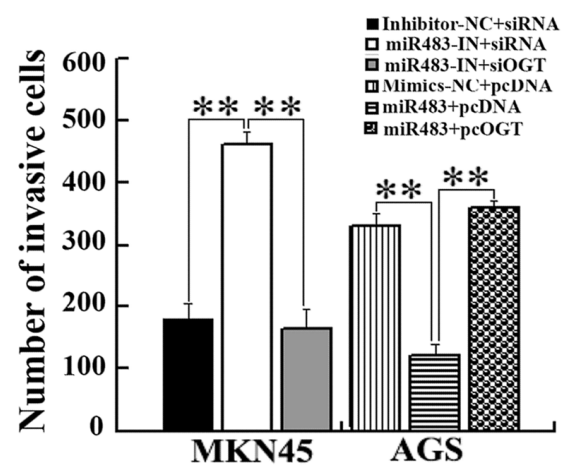

\section{B}
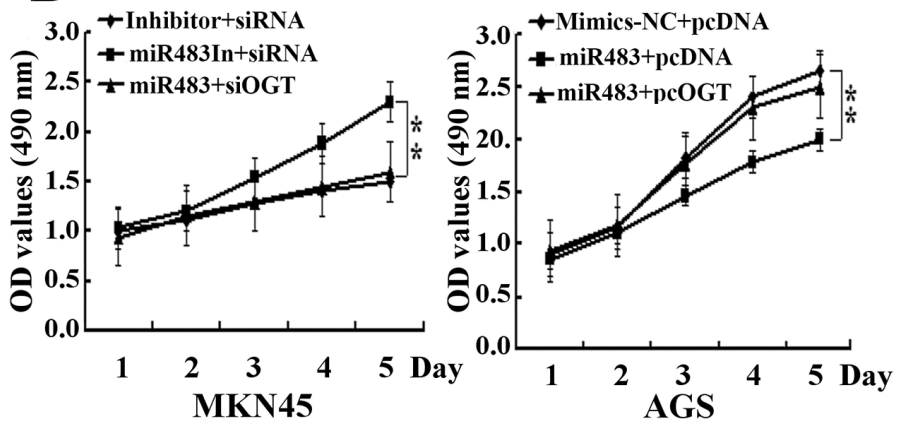

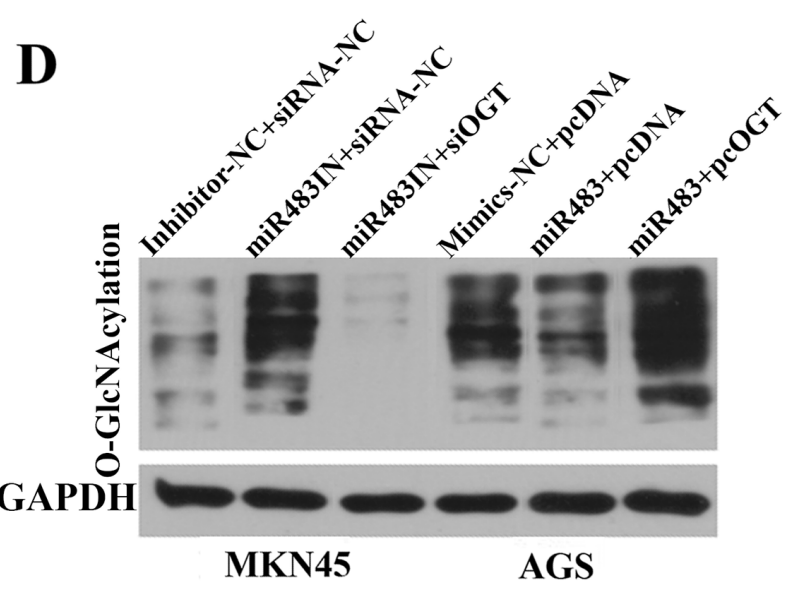

Figure 4. OGT is involved in miR-483 regulation of gastric cancer cell proliferation, invasion and protein O-GlcNAcylation. MKN45 cells were transfected with miR-483 inhibitor or co-transfected with miR-483 inhibitor and OGT specific siRNAs (siOGT), while AGS cells were transfected with miR-483 mimics or co-transfected with miR-483 mimics and OGT expression vector (pcOGT). (A) Western blotting analysis of OGT expression in MKN45 and AGS cells. (B) Determination of cell proliferation with the MTT assay. (C) Determination of cell invasion ability with the Transwell assay. (D) Determination of protein O-GlcNAcylation level by Western blot. ${ }^{\star} \mathrm{p}<0.05,{ }^{* *} \mathrm{p}<.01$ compared with control group. 
aerobic glycolysis [22-26]. OGT (O-GlcNAc transferase) uses UDP-GlcNAc as a substrate to add $\beta$ - $\mathrm{N}$-acetylglucosamine onto serine and threonine residues of a diverse range of nuclear and cytoplasmic proteins that are involved in various biological processes, such as transcription, translation, cell cycle, nutrient sensing and epigenetic regulation [27,28]. Consistent with these previous studies, we indicated that modulating expression of miR-483 could influence total protein O-GlcNAcylation levels in gastric cancer cells. OGT has been reported to consistently increase in a wide variety of human cancers including gastric cancer and is involved in the initiation and development of cancers by enhancing cell proliferation and invasion [29-31]. However, the mechanism through which OGT is maintained at a high expression level in cancer cells remains unknown. Our findings provide strong evidence that downregulation of miR-483 may be a potential mechanism for the elevated OGT expression in gastric cancer.

Our biological function studies further suggest that miR-483 represses gastric cancer proliferation and invasion mainly by targeting OGT. Moreover, we found that miR-483 can suppress total protein O-GlcNAcylation in gastric cancer cells through directly targeting OGT. Our data are consistent with previous studies which showed that down-regulation of OGT inhibited cell growth and invasion of gastric cancer cells in vitro $[32,33]$. Our results reveal that miR-483 is critical in controlling the protein O-GlcNAcylation levels and regulating the proliferation and invasion potential of gastric cancer cells. Therefore, our findings might provide a potential diagnostic marker and therapeutic target for gastric cancer.

\section{References}

[1] VAN CUTSEM E, SAGAERT X, TOPAL B, HAUSTERMANS K, PRENEN H. Gastric cancer. Lancet 2016; 388: 2654-2664. https://doi.org/10.1016/S0140-6736(16)30354-3

[2] DESANTIS CE, LIN CC, MARIOTTO AB, SIEGEL RL, STEIN KD et al. Cancer treatment and survivorship statistics, 2014. CA Cancer J Clin 2014; 64: 252-271. https://doi. org/10.3322/caac. 21235

[3] CHENDRIMADA TP, GREGORY RI, KUMARASWAMY E, NORMAN J, COOCH N et al. TRBP recruits the dicer complex to Ago2 for microRNA processing and gene silencing. Nature 2005; 436: 740-744. https://doi.org/10.1038/nature 03868

[4] HAYE J, PERUZZI PP, LAWLER S. MicroRNAs in cancer: biomarkers, functions and therapy. Trends Mol Med 2014; 20: 460-469. https://doi.org/10.1016/j.molmed.2014.06.005

[5] ZHANG L, HUANG Z, ZHANG H, ZHU M, ZHU W et al. Prognostic value of candidate microRNAs in gastric cancer: A validation study. Cancer Biomark 2017; 18: 221-230. https://doi.org/10.3233/CBM-160091

[6] YEPES S, LOPEZ R, ANDRADE RE, RODRIGUEZ-URREGO PA, LOPEZ-KLEINE L et al. Co-expressed miRNAs in gastric adenocarcinoma. Genomics 2016; 108: 93-101. https://doi.org/10.1016/j.ygeno.2016.07.002
[7] SHIN VY, NG EK, CHAN VW, KWONG A, CHU KM. A three-miRNA signature as promising non-invasive diagnostic marker for gastric cancer. Mol Cancer 2015; 14: 202. https://doi.org/10.1186/s12943-015-0473-3

[8] FU H, TIE Y, XU C, ZHANG Z, ZHU J et al. Identification of human fetal liver miRNAs by a novel method. FEBS Lett 2005; 579: 3849-3854. https://doi.org/10.1016/j.febslet.2005.05.064

[9] LANDGRAF P, RUSU M, SHERIDAN R, SEWER A, IOVINO $\mathrm{N}$ et al. A mammalian microRNA expression atlas based on small RNA library sequencing. Cell 2007; 129: 1401-1414. https://doi.org/10.1016/j.cell.2007.04.040

[10] CAI H, XU J, HAN Y, LU Z, HAN T et al. Integrated miRNArisk gene-pathway pair network analysis provides prognostic biomarkers for gastric cancer. Onco Targets Ther 2016; 19: 2975-2986. https://doi.org/10.2147/OTT.S95129

[11] LEE SW, PARK KC, KIM JG, MOON SJ, KANG SB et al. Dysregulation of MicroRNA-196b-5p and MicroRNA-375 in Gastric Cancer. J Gastric Cancer 2016; 16: 221-229. https:// doi.org/10.5230/jgc.2016.16.4.221

[12] ASSUMPCAO MB, MOREIRA FC, HAMOY IG, MAGALHAES L, VIDAL A et al. High-Throughput miRNA Sequencing Reveals a Field Effect in Gastric Cancer and Suggests an Epigenetic Network Mechanism. Bioinform Biol Insights 2015; 9: 111-11. https://doi.org/10.4137/BBI.S24066

[13] WANG Z, WANG J, YANG Y, HAO B, WANG R et al. Loss of has-miR-337-3p expression is associated with lymph node metastasis of human gastric cancer. J Exp Clin Cancer Res 2013; 32: 76. https://doi.org/10.1186/1756-9966-32-76

[14] WANG C, SUN Y, WU H, YU S, ZHANG L et al. Elevated miR-483-3p expression is an early event and indicates poor prognosis in pancreatic ductal adenocarcinoma. Tumour Biol 2015; 36: 9447-9456. https://doi.org/10.1007/s13277015-3690-x

[15] SONG Q, XU Y, YANG C, CHEN Z, JIA C et al. miR-483-5p promotes invasion and metastasis of lung adenocarcinoma by targeting RhoGDI1 and ALCAM. Cancer Res 2014; 74: 3031-3042. https://doi.org/10.1158/0008-5472.CAN-132193

[16] BI C, CHUNG TH, HUANG G, ZHOU J, YAN J et al. Genome-wide pharmacologic unmasking identifies tumor suppressive microRNAs in multiple myeloma. Oncotarget 2015; 6: 26508-26518. https://doi.org/10.18632/oncotarget.4769

[17] BERTERO T, BOURGET-PONZIO I, PUISSANT A, LOUBAT A, MARI B et al. Tumor suppressor function of miR483-3p on squamous cell carcinomas due to its pro-apoptotic properties. Cell Cycle 2013; 12: 2183-2193. https://doi. org/10.4161/cc.25330

[18] ZHANG Y, ZHANG HE, LIU Z. MicroRNA-147 suppresses proliferation, invasion and migration through the AKT/ mTOR signaling pathway in breast cancer. Oncol Lett 2016; 11: 405-410. https://doi.org/10.3892/ol.2015.3842

[19] WANG N, ZHAN T, KE T, HUANG X, KE D et al. Increased expression of RRM2 by human papillomavirus E7 oncoprotein promotes angiogenesis in cervical cancer. Br J Cancer 2014; 110: 1034-1044. https://doi.org/10.1038/bjc.2013.817 
[20] GATENBY RA, GILLIES RJ. Why do cancers have high aerobic glycolysis? Nat Rev Cancer 2004; 4: 891-899. https://doi. org/10.1038/nrc1478

[21] JONES RG, THOMPSON CB. Tumor suppressors and cell metabolism: a recipe for cancer growth. Gene Dev 2009; 23: 537-548. https://doi.org/10.1101/gad.1756509

[22] FERRER CM, LYNCH TP, SODI VL, FALCONE JN, SCHWAB LP et al. O-GlcNAcylation regulates cancer metabolism and survival stress signaling via regulation of the HIF-1 pathway. Mol Cell 2014; 54: 820-831. https://doi. org/10.1016/j.molcel.2014.04.026

[23] ITKONEN HM, GORAD SS, DUVEAU DY, MARTIN SE, BARKOVSKAYA A et al. Inhibition of O-GlcNAc transferase activity reprograms prostate cancer cell metabolism. Oncotarget 2016; 7: 12464-12476. https://doi.org/10.18632/oncotarget.7039

[24] FERRER CM, REGINATO MJ. Cancer metabolism: cross talk between signaling and O-GlcNAcylation. Methods Mol Biol 2014; 1176: 73-88. https://doi.org/10.1007/978-1-49390992-6_7

[25] ONODERA Y, NAM JM, BISSELL MJ. Increased sugar uptake promotes oncogenesis via EPAC/RAP1 and O-GlcNAc pathways. J Clin Invest 2014; 124: 367-384. https://doi. org/10.1172/JCI63146

[26] FERRER CM, LU TY, BACIGALUPA ZA, KATSETOS CD, SINCLAIR DA et al. O-GlcNAcylation regulates breast cancer metastasis via SIRT1 modulation of FOXM1 pathway. Oncogene 2017; 36: 559-569. https://doi.org/10.1038/ onc. 2016.228

[27] HART GW, HOUSLEY MP, SLAWSON C. Cycling of Olinked beta-N-acetylglucosamine on nucleocytoplasmic proteins. Nature 2007; 446: 1017-1022. https://doi.org/10.1038/ nature 05815
[28] RUAN HB, NIE Y, YANG X. Regulation of protein degradation by O-GlcNAcylation: crosstalk with ubiquitination. Mol Cell Proteomics 2013; 12: 3489-3497. https://doi. org/10.1074/mcp.R113.029751

[29] JIANG M, QIU Z, ZHANG S, FAN X, CAI X et al. Elevated O-GlcNAcylation promotes gastric cancer cells proliferation by modulating cell cycle related proteins and ERK $1 / 2$ signaling. Oncotarget 2016; 7: 61390-61402. https://doi. org/10.18632/oncotarget.11359

[30] JANG TJ, KIM UJ. O-GlcNAcylation is associated with the development and progression of gastric carcinoma. Pathol Res Pract 2016; 212: 622-630. https://doi.org/10.1016/j. prp.2016.04.002

[31] YANG Y, YIN X, YANG H, XU Y. Histone demethylase LSD2 acts as an E3 ubiquitin ligase and inhibits cancer cell growth through promoting proteasomal degradation of OGT. Mol Cell 2015; 58: 47-59. https://doi.org/10.1016/j. molcel.2015.01.038

[32] WEN T, HOU K, LI Z, LI L, YU H et al. Silencing $\beta$-linked Nacetylglucosamine transferase induces apoptosis in human gastric cancer cells through PUMA and caspase-3 pathways. Oncol Rep 2015; 34: 3140-3146. https://doi.org/10.3892/ or.2015.4276

[33] CHENG S, REN J, SU L, LIU J, LIU Q et al. O-GlcNAcylation of the Signaling Scaffold Protein, GNB2L1 Promotes its Degradation and Increases Metastasis of Gastric Tumours. Biochem Biophys Res Commun 2016; 478: 1497-1502. https:// doi.org/10.1016/j.bbrc.2016.08.074 